\title{
Defining the contributions of network clock models to millisecond timing
}

\author{
Uma R. Karmarkar* \\ Harvard Business School, Boston, MA USA \\ *Correspondence: ukarmarkar@hbs.edu
}

Our ability to measure time extends from microseconds to days (Buonomano and Karmarkar, 2002; Buhusi and Meck, 2005). Given the wealth of experimental support for a number of different models of timing, it has been recently suggested that multiple mechanisms act in concert to transition smoothly between both temporal ranges and modalities (Wiener et al., 2011). This underscores the importance of determining the specific contributions of individual mechanisms to better understand these transitions and any relevant idiosyncrasies of timing in a particular context.

We focus here on interval discrimination in the range of tens to hundreds of milliseconds, which plays an important role in a variety of tasks, such as speech processing, motion detection, and fine motor coordination. It has been proposed that such timing can emerge directly from the temporal properties intrinsic to neural circuits (Buonomano and Mauk, 1994; Buonomano and Merzenich, 1995; Karmarkar and Buonomano, 2007; for review see Ivry and Schlerf, 2008). Broadly, this class of mechanisms can be described as population or network clocks, as the timing of incoming stimuli is coded as the changes they effect in a population of neurons. This can also be thought of as the change in the overall state of the network. Compared to models of millisecond timing based on a single centralized mechanism (Treisman, 1963; Church, 1984; Gibbon et al., 1997; Ivry and Spencer, 2004) network clock models imply that timing is being done in multiple loci across the cortex. This is interesting because it means that the dynamics of timing are dependent on the properties of the underlying local circuitry, which could vary across modalities.

To understand the behavior of network clocks, it is useful to examine a particular instantiation, referred to as a state-dependent network (SDN), which has been developed in the context of sensory processing (Karmarkar and Buonomano, 2007). For SDNs an incoming stimulus changes the network state not only by causing some population of neurons to fire, but also by engaging a number of intrinsic properties such as short-term plasticity, that change with specific time constants on the millisecond scale. As a result, the response of the network to a particular piece of temporal information is dependent on its recent history. Thus instead of marking each interval separately, timing is done continuously, with the network linking multiple signals together as a temporal object. The SDN can only measure information independently, or reset, when the interval between stimuli has been sufficiently long to allow the network to return to its baseline state. This makes two predictions about interval discrimination, the first being that a variable context (or "distractor" stimulus) should have a greater impact or disruption on timing than a fixed one. The second prediction is that when comparing two intervals, timing of the second will be influenced by the first if they are not separated by more time than the reset threshold. These predictions can be tested psychophysically to distinguish the extent to which various types of timing arise from network clocks.

In the auditory system, multiple studies examining interval discrimination with reset tasks have shown results consistent with an SDN model (Karmarkar and Buonomano, 2007; Buonomano et al., 2009; Spencer et al., 2009). Based on this data, the influence of the SDN appears to fall off somewhere between intervals above 250 but below 500 ms (Buonomano et al., 2009). This limit is potentially as restrictive as $300 \mathrm{~ms}$, since stimuli including that interval do not show the expected pattern of impairment due to a variable context (Spencer et al., 2009). However, a secondary analysis of the data from that study might suggest that distractor signals prior to the target interval do appear to exert some bias on discrimination as long as the whole stimulus sequence, or temporal object, does not significantly exceed $400 \mathrm{~ms}$ (Figures 3 and 5,
Spencer et al., 2009). A threshold in this vicinity is consistent with the assumptions of the SDN model since it is dependent on the time constants of short-term plasticity, which are on the order of a few hundreds of milliseconds (e.g. Zucker, 1989).

Such a restriction in range could be perceived as a challenge to the relevance of SDNs, or network clocks in general, to other sensory modalities. Though intervals of less than 300-400 ms can be useful for somatosensory timing, the visual system appears to operate on a fundamentally slower timescale. Experiments in which individuals had to reproduce durations represented by visual stimuli showed that participants' lowest estimated duration was $300 \mathrm{~ms}$, even when the target interval was less than $100 \mathrm{~ms}$, suggesting difficulty in accurately perceiving those shorter times (Lewis and Miall, 2009). In addition, visual discrimination of intervals has been shown to be less precise, that is, to have a higher variance, than auditory perception of the same durations (Merchant et al., 2008), which could prevent effective measurement of time spans less than $200 \mathrm{~ms}$. Based on this data, the range over which the SDN operates for auditory stimuli might appear to render it inconsequential for visual ones.

It should be noted though, that previous studies have revealed some visual discriminatory capabilities for intervals in the 100 to 200-ms range (e.g. Mattes and Ulrich, 1998; Westheimer, 1999). Furthermore, interval timing in the visual system is spatially localized (Johnston et al., 2006; Burr et al., 2007), suggesting that the mechanism is specific to early visual cortices, consistent with the idea of a local network clock. As such, it is possible that some of the reduction in precision for interval timing is due to the inherent variance of the basic response time in primary visual cortex. This could be considered a timeindependent issue that influences the system by adding noise rather than indicating a non-SDN mechanism. 
Vision has been considered a difficult modality for defining or studying temporal processing mechanisms. This is because visual timing is extremely sensitive to a number of atemporal stimulus characteristics (Eagleman, 2008), and is generally tightly linked to spatial information, as in motion detection. However, we propose that it is key in determining whether the SDN can be considered a general model of sensory timing.

Finally, it is important to recognize that there are other network clock models based on differing circuitry (e.g. Buonomano and Mauk, 1994; Medina et al., 2000; Fiete et al., 2010). This reflects the broader concept of an intrinsic timer, that temporal processing is dependent on the specific properties of the neural locus in question. The differences in the structure of these models also lead to differences in the range of times they can process, and their ability to integrate spatial and temporal information. For example, it has been proposed that population clock models that leverage recurrent excitatory connections with strong weights can account for motor timing that extends from milliseconds into seconds (Buonomano and Laje, 2010). Despite this diversity, network clock models largely show the same phenotype of continuous temporal processing, in which sequences are treated as temporal objects, making reset-type tasks a general diagnostic tool for this class of timers. As a result, using psychophysical measures to investigate timing directly across modalities is an important first step in defining the contribution of network clock models to human interval discrimination.

\section{REFERENCES}

Buhusi, C. V., and Meck, W. H. (2005). What makes us tick? Functional and neural mechanisms of interval timing. Nat. Rev. Neurosci. 6, 755-765.

Buonomano, D. V., Bramen, J., and Khodadadifar, M. (2009). Influence of the interstimulus interval on temporal processing and learning: testing the statedependent model. Philos. Trans. R. Soc. Lond. B Biol. Sci. 364, 1865-1873.

Buonomano, D. V., and Karmarkar, U. R. (2002). How do we tell time? Neuroscientist 8, 42-51.

Buonomano, D.V., and Laje, R. (2010). Population clocks: motor timing with neural dynamics. Trends Cogn. Sci. (Regul. Ed.) 14, 520-527.

Buonomano, D. V., and Mauk, M. D. (1994). Neural network model of the cerebellum: temporal discrimination and the timing of motor responses. Neural Comput. 6, 38-55.

Buonomano, D. V., and Merzenich, M. M. (1995) Temporal information transformed into a spatial code by a neural network with realistic properties. Science 267, 1028-1030.

Burr, D., Tozzi, A., and Morrone, M. C. (2007). Neural mechanisms for timing visual events are spatially selective in real-world coordinates. Nat. Neurosci. 10, 423-425.

Church, R.M. (1984). "Properties of the internal clock," in Annals of the New York Academy of Sciences, Vol. 423, Timing and Time Perception, eds J. Gibbon and L. Allan (New York: New York Academy of Sciences), 556-582.

Eagleman, D. M. (2008). Human time perception and its illusions. Curr. Opin. Neurobiol. 18, 131-136.

Fiete, I. R., Senn, W., Wang, C. Z. H., and Hahnloser, R. H. R. (2010). Spike-time-dependent plasticity and heterosynaptic competition organize networks to produce long scale-free sequences of neural activity. Neuron 65, 563-576.

Gibbon, J., Malapani, C., Dale, C. L., and Gallistel, C. R. (1997). Toward a neurobiology of temporal cognition: advances and challenges. Curr. Opin. Neurobiol. 7, 170-184.

Ivry, R. B., and Schlerf, J.E. (2008). Dedicated and intrinsic models of time perception. Trends Cogn. Sci. (Regul. Ed.) 12, 273-280.

Ivry, R. B., and Spencer, R. M. C. (2004). The neural representation oftime. Curr. Opin. Neurobiol. 14, 225-232.
Johnston, A., Arnold, D. H., and Nishida, S. (2006). Spatially localized distortions of event time. Curr. Biol. 16, 472-479.

Karmarkar, U. R., and Buonomano, D.V. (2007). Timing in the absence of clocks: encoding time in neural network states. Neuron 53, 427-438.

Lewis, P.A., and Miall, R. C. (2009). The precision of temporaljudgement: milliseconds, many minutes, and beyond. Philos. Trans. R. Soc. Lond. B Biol. Sci. 364, 1897-1905.

Mattes, S., and Ulrich, R. (1998). Directed attention prolongs the perceived brief duration of a stimulus. Percept. Psychophys. 60, 1305-1317.

Medina, J. F., Garcia, K. S., Nores, W. L., Taylor, N. M., and Mauk, M. D. (2000). Timing mechanisms in the cerebellum: testing predictions of a large-scale computer simulation. J. Neurosci. 20, 5516-5525.

Merchant, H., Zarco, W., and Prado, L. (2008). Do we have a common mechanism for measuring time in the hundreds of millisecond range? Evidence from multipleinterval timing tasks. J. Neurophysiol. 99, 939-949.

Spencer, R. M. C., Karmarkar, U. R., and Ivry, R. B. (2009). Evaluating dedicated and intrinsic models of temporal encoding by varying context. Philos. Trans. R. Soc. Lond. B Biol. Sci. 364, 1853-1863.

Treisman, M. (1963). Temporal discrimination and the indifference interval: implications for a model of the “internal clock.” Psychol. Monogr. 77, 1-31.

Westheimer, G. (1999). Discrimination of short time intervals by the human observer. Exp. Brain Res. 129, 121-126.

Wiener, M., Matell, M. S., and Coslett, H. B. (2011). Multiple mechanisms for temporal processing. Front Integr. Neurosci. 5:31. doi: 10.3389/fnint.2011.00031

Zucker, R. S. (1989). Short-term synaptic plasticity. Annu. Rev. Neurosci. 12, 13-31.

Received: 01 August 2011; accepted: 01 August 2011; published online: 18 August 2011.

Citation: Karmarkar UR (2011) Defining the contributions of network clock models to millisecond timing. Front. Integr. Neurosci. 5:41. doi: 10.3389/fnint.2011.00041

Copyright $(\odot 2011$ Karmarkar. This is an open-access article subject to a non-exclusive license between the authors and Frontiers Media SA, which permits use, distribution and reproduction in other forums, provided the original authors and source are credited and other Frontiers conditions are complied with. 